\title{
Sniff test: Does what we measure at the nose reflect what happens in the chest wall?
}

The strength of respiratory muscles is measured in terms of pressure, being the gradient that moves flow/volume to ventilate the lungs.

Sniff test is a technically simple voluntary test that quantifies the strength of inspiratory muscles. Sniff nasal inspiratory pressure (SNIP) ${ }^{1}$ is a non-invasive parameter extensively used in the clinical, being considered more natural and pleasant than maximal inspiratory pressure measurements. It solves the possible leak problems of the use of a mouthpiece, particularly in neuromuscular patients. ${ }^{2,3}$ In amyotrophic lateral sclerosis, for example, SNIP is a predictor of survival and tracheostomy. ${ }^{4,5}$

SNIP is used both as a global indicator of inspiratory muscle strength, being considered a good surrogate of oesophageal pressure, but also as specific index of diaphragmatic force, when associated to trans-diaphragmatic pressure measurement. In order to discern the relative contribution of each major respiratory muscle to SNIP, the use of transoesophageal catheters is required, but they are not always well tolerated or routinely adopted in clinical practice or they can be problematic in presence of important bulbar implications.

Alternately, the relative contributions of various respiratory muscles can be derived by the analysis of thoracoabdominal volume variations, normally assessed by non-invasive methods. ${ }^{6,7}$

A pure contraction of the diaphragm results into abdominal expansion, while the thorax moves inwardly. By contrast, a pure contraction of inspiratory ribcage muscles makes the thorax expandwith theabdomen moving inwardly(Figure 1A). Paradoxical movements are consequences of the lack of the counteracting action of respiratory muscles to pleural pressure becoming subatmospheric. ${ }^{8-10}$ In terms of thoracoabdominal contribution to total chest wall expansions, paradoxical movements result into a negative contribution of the compartment that moves paradoxically, while the other compartment contributes $>100 \%$ to compensate (Figure 1B).

In order to verify if and how SNIP varied according to thoracoabdominal volume variations in 28 candidates for lung transplant (median age: 42 years; median FVC: $47.5 \%$ predicted; median $\mathrm{FEV}_{1}: 30 \%$ predicted), we measured the abdominal volume percentage contribution, using optoelectronic plethysmography (OEP System; BTS, Milan, Italy), ${ }^{7}$ during awake quiet breathing $\left(\mathrm{AB}_{\mathrm{QB}}\right)$ and sniff manoeuvres $\left(\mathrm{AB}_{\mathrm{SN}}\right)$ in supine position. SNIP was measured simultaneously using a Respiratory Pressure Meter [MicroMedical MicroRPM 01 (Respiratory Pressure Meter)] following standard international guidelines. ${ }^{1}$ After a period of at least 5 minutes of quiet breathing, patients, who were all familiar with respiratory tests, were asked to perform maximal sniffs.

Sixty eight sniff manoeuvres were analysed and classified into four groups according to $\mathrm{AB}_{\mathrm{SN}}$ (Figure 1A-B). Sixteen patients showed thoracic paradox (parTH) defined as $\mathrm{AB}_{\mathrm{SN}}>100 \%, 24$ patients showed predominantly abdominal pattern $(\mathrm{AB})$, define as $50 \%<\mathrm{AB}_{\mathrm{SN}} \leq 100 \%, 16$ patients were predominantly thoracic $(\mathrm{TH})$ defined as $0 \% \leq \mathrm{AB}_{\mathrm{SN}} \leq 50 \%$ and 12 patients had abdominal paradox (parAB) defined as $\mathrm{AB}_{\mathrm{SN}}<0 \%$.

By definition, $\mathrm{AB}_{\mathrm{SN}}$ was different $(P<0.001)$ among the four groups, whereas SNIP (median: $53 \mathrm{cmH}_{2} \mathrm{O}, P=0.792$; Figure $1 \mathrm{C}$ ) and $\mathrm{AB}_{\mathrm{QB}}$ (median: 75.2\%; $P=0.373$; Figure 1D) did not differ.

SNIP per se could not discriminate between thoracic or diaphragmatic manoeuvre, in spite of evident different thoracoabdominal patterns that might be associated to different relative contribution of the major inspiratory muscles. In the majority of the cases, the manoeuvre was completely (parTH) or predominantly $(\mathrm{AB})$ accomplished by the diaphragm, otherwise sniff was driven by inspiratory ribcage muscles (RC and parAB). parTH and parAB were characterised by paradoxical inward movements and for this reason, they might indicate severe weakness or paralysis of the inspiratory ribcage muscles or of the diaphragm, respectively. The analysis during quite breathing revealed, as expected in supine position, the diaphragm to lead inspiration with no paradoxical thoracic motion (Figure 1D). ${ }^{7} \mathrm{AB}_{\mathrm{SN}}$, therefore, varied because of the strategies adopted by the patients to interpret and perform the manoeuvre and not for the presence of specific inspiratory muscles involvement.

Although these conclusions need to be confirmed by bigger studies on different populations and postures, our preliminary data arose important clinical considerations:

1. there is the risk that SNIP masks the weakness progression of a specific group of inspiratory muscles; 
(A)

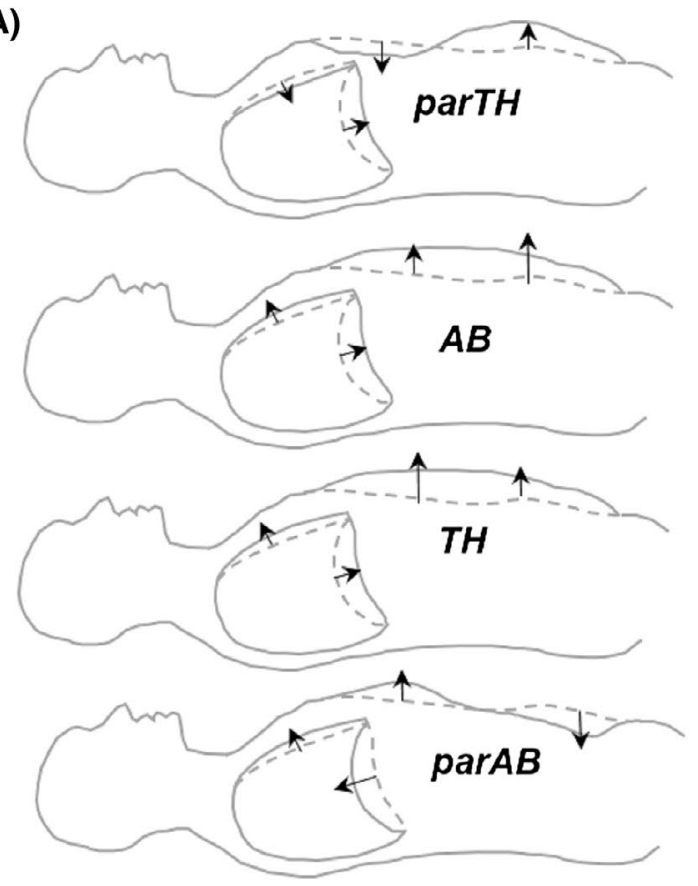

(B)

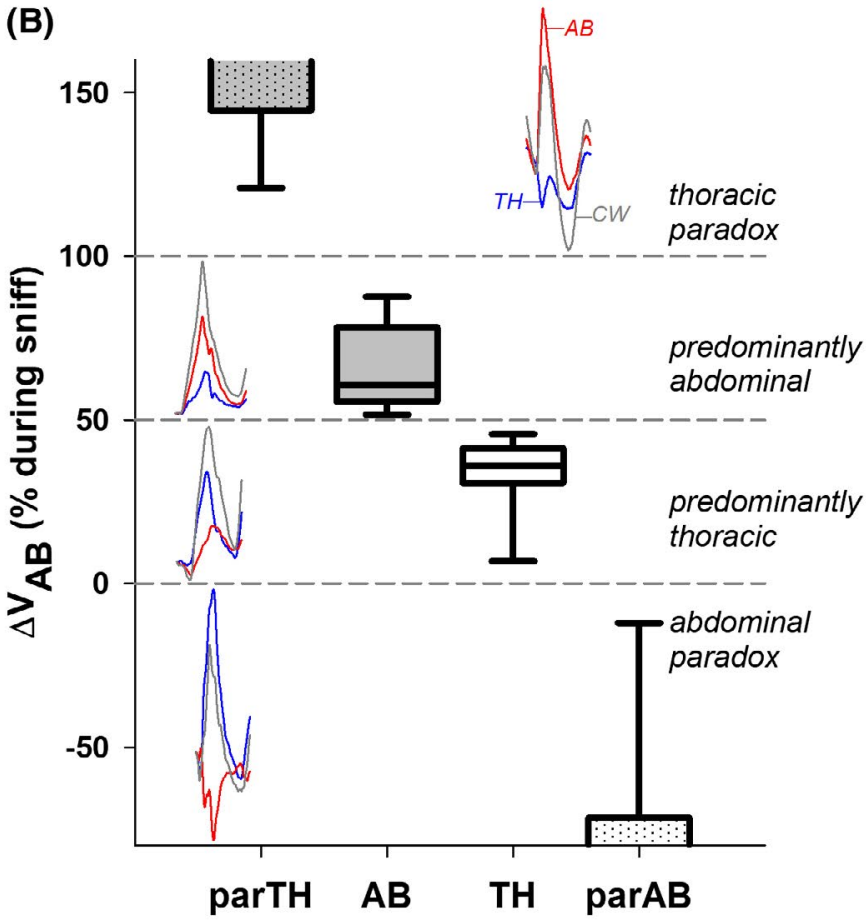

(D)

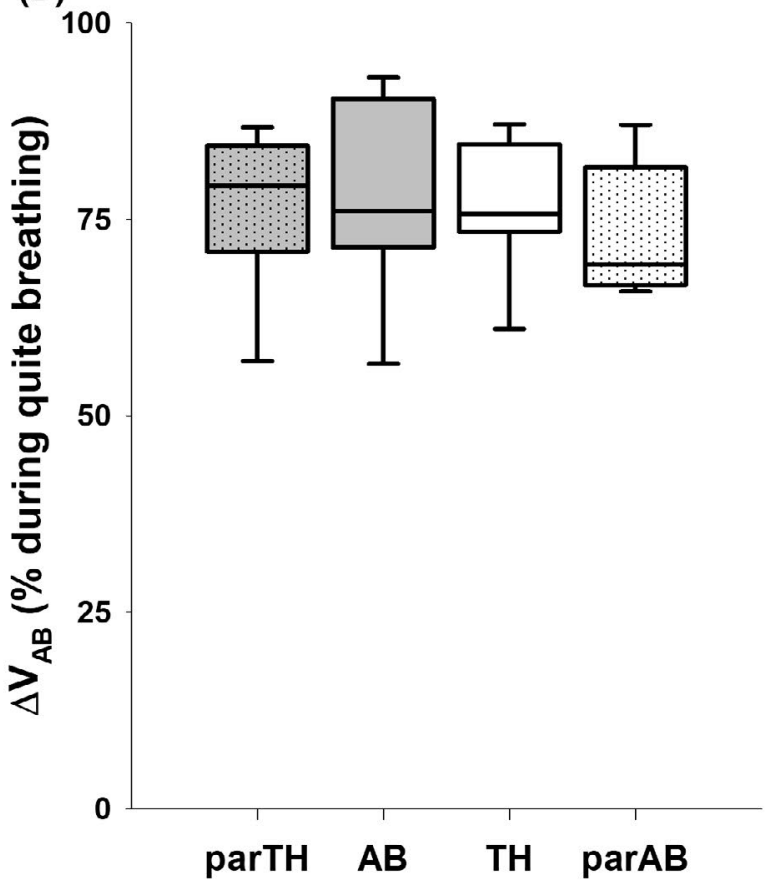

(C)

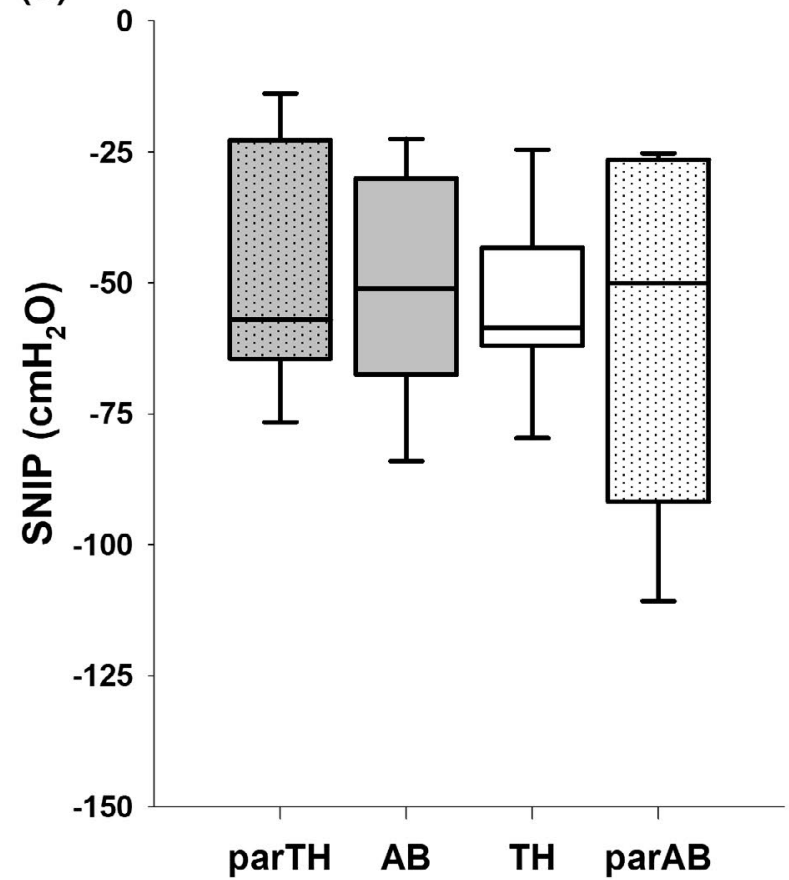

F I G URE 1 (a) schematic representation of the four considered thoracoabdominal strategies. From top to bottom: presence of thoracic paradox (parTH), manoeuverer predominantly abdominal (AB), manoeuverer predominantly thoracic (TH) and presence of abdominal paradox (parAB). The arrows indicate the direction of the movement; (b) supine abdominal volume percentage contribution during sniff manoeuvres in the four considered thoracoabdominal strategies. Representative cases of thoracic (blue line), abdominal (red line) and chest wall (grey line) volume changes during sniff manoeuvre are reported. In case of paradoxical movements (top and bottom panels), the compartment that moves paradoxically contributes in a negative way (because it reduces) and as a consequence, the other compartment exceeds the expansion of the chest wall, being the sum of the thoracoabdominal contributions; (c) sniff nasal inspiratory pressure (SNIP) during sniff manoeuvres in the four considered thoracoabdominal strategies; (d) supine abdominal volume percentage contribution during awake quiet breathing before sniff manoeuvre 
2. there can be the need to standardise sniff performance by instructing patients to use mainly the diaphragm or inspiratory ribcage muscles and to separately collect the two maximal corresponding SNIP values for each strategy;

3. combining the analysis of thoracoabdominal motion during sniff might add important non-invasive indication on the relative contribution of the major inspiratory muscles, with SNIP becoming a more specific index.

The extent of the contribution of each inspiratory muscle group is important in the interpretation of sniff test; otherwise SNIP could be clinically insensitive to serious loss of function of individual inspiratory muscles. This might be also extended to maximal inspiratory and expiratory pressures.

\section{ACKNOWLEDGEMENT}

Special thanks go to Gabriele Bardi, Matteo Cagnetta and Edoardo Cornalba for the help in the preliminary analysis of the data.

This work was conducted without funds.

\section{CONFLICT OF INTERESTS}

The authors have stated explicitly that there are no conflicts of interest in connection with this article.

\section{AUTHOR CONTRIBUTIONS}

Designed research/study: ALM, EP and AP; Performed research/study: ALM and EP; Analysed data: ALM; Wrote the paper: ALM; Revised the paper: all the authors.

\author{
Antonella LoMauro ${ }^{1}$ (D) \\ Emilia Privitera $^{2}$ \\ Andrea Aliverti ${ }^{1}$ iD \\ Mario Nosotti ${ }^{2,3}$ \\ Alessandro Palleschi ${ }^{2}$ \\ ${ }^{1}$ Dipartimento di Elettronica, Informazione e \\ Bioingegneria, Politecnico di Milano, Milan, Italy \\ ${ }^{2}$ Thoracic Surgery and Lung Transplantation Unit, \\ Fondazione IRCCS Ca' Granda-Ospedale Maggiore \\ Policlinico of Milan, Milan, Italy
}

${ }^{3}$ Department of Pathophysiology and Transplantation,
Surgical and Dental Sciences, Università degli Studi
di Milano, Milan, Italy

Correspondence

Antonella LoMauro, Dipartimento di Elettronica, Informazione e Bioingegneria, Politecnico di Milano, piazza Leonardo Da Vinci, 20133 Milan, Italy. Email: antonella.lomauro@polimi.it

\section{ORCID}

Antonella LoMauro (D) https://orcid. org/0000-0002-1408-6971

Andrea Aliverti (D) https://orcid.org/0000-0002-2950-0231

\section{REFERENCES}

1. Laveneziana P, Albuquerque A, Aliverti A, et al. ERS statement on respiratory muscle testing at rest and during exercise. Eur Respir J. 2019;53:1801214.

2. Nicot F, Hart N, Forin V, et al. Respiratory muscle testing: a valuable tool for children with neuromuscular disorders. Am J Respir Crit Care Med. 2006;174:67-74.

3. Fauroux B, Aubertin G, Cohen E, Clément A, Lofaso F. Sniff nasal inspiratory pressure in children with muscular, chest wall or lung disease. Eur Respir J. 2009;33:113-117.

4. Capozzo R, Quaranta VN, Pellegrini F, et al. Sniff nasal inspiratory pressure as a prognostic factor of tracheostomy or death in amyotrophic lateral sclerosis. J Neurol. 2015;262:593-603.

5. Morgan RK, McNally S, Alexander M, et al. Use of Sniff nasalinspiratory force to predict survival in amyotrophic lateral sclerosis. Am J Respir Crit Care Med. 2005;171:269-274.

6. Zimmerman PV, Connellan SJ, Middleton HC, et al. Postural changes in rib cage and abdominal volume-motion coefficients and their effect on the calibration of a respiratory inductance plethysmograph. Am Rev Respir Dis. 1983;127:209-214.

7. Romei M, Mauro AL, D'angelo MG, et al. Effects of gender and posture on thoraco-abdominal kinematics during quiet breathing in healthy adults. Respir Physiol Neurobiol. 2010;172:184-191.

8. Macklem PT. Respiratory muscles: the vital pump. Chest. 1980;78:753-758.

9. Troyer AD, Wilson TA. Action of the diaphragm on the rib cage. J Appl Physiol. 2016;121:391-400.

10. De Troyer A, Kirkwood PA, Wilson TA. Respiratory action of the intercostal muscles. Physiol Rev. 2005;85:717-756. 